\title{
Point-of-care testing of capillary glucose in the exclusion and diagnosis of diabetes in remote Australia
}

\author{
Julia V Marley, Stephanie Davis, Kerryn Coleman, Bradleigh D Hayhow, Greg Brennan, Jacki K Mein, Carmel Nelson, \\ David Atkinson and Graeme P Maguire
}

$\mathrm{D}$ iabetes and its associated complications are a significant health problem facing Indigenous Australians. The prevalence of type 2 diabetes mellitus in Aboriginal and Torres Strait Islander peoples has been estimated to be as high as $32 \%^{1,2}$ While early diagnosis and treatment leads to improved outcomes, diabetes remains undiagnosed in up to $50 \%$ of patients. ${ }^{3-5}$

In the remote Kimberley region of Western Australia, guidelines for the diagnosis of diabetes, based on national Australian guidelines, ${ }^{6,7}$ require two separate laboratory venous blood glucose measurements showing raised levels in an asymptomatic patient, one elevated venous blood glucose measurement in a symptomatic patient, or an oral glucose tolerance test consistent with diabetes. ${ }^{8}$ Due to the distance of many health care clinics from a laboratory, and the infrequent transport links, the requirement for venous glucose results can significantly delay diagnosis, patient feedback and education. Many people also move between communities and venous glucose results may not be readily available when the person is next seen.

Immediate results with diagnostic significance would improve the diagnosis and management of diabetes at remote sites. While earlier studies have shown a high level of concordance between laboratory venous blood glucose and point-of-care (POC) capillary blood glucose measurements, these have mainly been conducted in controlled, urban environments. ${ }^{9-11}$ In 2003, Martin et al, ${ }^{2}$ at a single remote clinic in the Kimberley, showed that POC capillary glucose measurement may be useful as a screening tool to exclude a diagnosis of diabetes. However, this study used well calibrated and maintained equipment operated by specifically trained staff, and thus did not reflect "real world" remote health care.

We aimed to determine whether POC capillary blood glucose measurement was sufficiently accurate to allow the diagnosis or exclusion of diabetes as part of usual remote primary health care practice.

\section{METHODS}

Data were collected by local health care providers from May to November 2006 at seven

\section{ABSTRACT}

Objectives: To determine the utility of point-of-care (POC) capillary blood glucose measurements in the diagnosis and exclusion of diabetes in usual practice in primary health care in remote areas.

Design: Cross-sectional study comparing POC capillary glucose results with corresponding venous glucose levels measured in a reference laboratory.

Participants: 200 participants aged 16-65 years enrolled: 198 had POC capillary glucose measurements; 164 also had acceptable venous glucose laboratory results.

Setting: Seven health care sites in the Kimberley region of Western Australia from May to November 2006.

Main outcome measures: Concordance and mean differences between POC capillary blood glucose measurement and laboratory measurement of venous blood glucose level; POC capillary blood glucose equivalence values for excluding and diagnosing diabetes, and their sensitivity, specificity and positive-predictive value.

Results: The concordance between POC and laboratory results was high ( $\rho=0.93$, $P<0.001)$. The mean difference in results was $0.48 \mathrm{mmol} / \mathrm{L}(95 \% \mathrm{Cl}, 0.23-0.73$; limits of agreement, -2.6 to $3.6 \mathrm{mmol} / \mathrm{L})$. The POC capillary glucose equivalence values for excluding and diagnosing diabetes were $<5.5 \mathrm{mmol} / \mathrm{L}$ (sensitivity, $53.3 \%$; specificity, 94.4\%; positive-predictive value, $88.9 \%$; for a venous value of $<5.5 \mathrm{mmol} / \mathrm{L}$ ) and $\geqslant 12.2 \mathrm{mmol} / \mathrm{L}$ (sensitivity, $83.3 \%$; specificity, $99.3 \%$; positive-predictive value, $95.2 \%$; for a venous value of $\geqslant 11.1 \mathrm{mmol} / \mathrm{L}$ ), respectively. While the choice of glucometer and whether or not patients were fasting altered these results, they did not have a significant influence on the diagnostic utility of POC glucose measurement in this setting.

Conclusion: POC capillary blood glucose analysers can be used as part of the process of diagnosing and excluding diabetes in remote rural communities using these locally established capillary equivalence values.

sites across the Kimberley (Box 1). Any patient who had venous blood glucose measurement requested as part of usual clinical care was invited to participate.

\section{POC glucose measurement and venipuncture}

POC measurement of capillary blood glucose and venipuncture were done concurrently and the time of each test was recorded. Data were excluded if POC capillary glucose measurement and venipuncture occurred more than 30 minutes apart.

Capillary blood glucose was measured by a primary health care provider via a fingerprick blood sample and analysed on the POC glucometer usually used at each site. No attempt was made to standardise health-provider education, or glucometer maintenance or calibration. The two glucometers used in our study were the Accu-Chek Advantage
(Roche Diagnostics, Sydney, NSW), which displays whole blood capillary glucose levels, and the MediSense Optium (Abbott Diabetes Care, Melbourne, Vic), which mathematically converts a whole blood measurement to a capillary plasma glucose level (technical data supplied by the manufacturer).

Venous whole blood samples were collected in containers with fluoride oxalate. Normal procedures at each clinic were used for storage (whole blood was stored at $4^{\circ} \mathrm{C}$ ) and transport of whole blood samples to one of the three laboratories in the Kimberley region (Box 1).

Venous plasma glucose levels were measured enzymatically on a Vitros 250 Analyser (Ortho-Clinical Diagnostics, Rochester, NY, USA) using glucose oxidase spectrophotometric dry chemistry. The distance to the laboratory from the study sites ranged from $2 \mathrm{~km}$ by road to $280 \mathrm{~km}$ by air, and the time 


\section{DIABETES}

1 Health care sites where the study was conducted, the Kimberley, Western Australia*

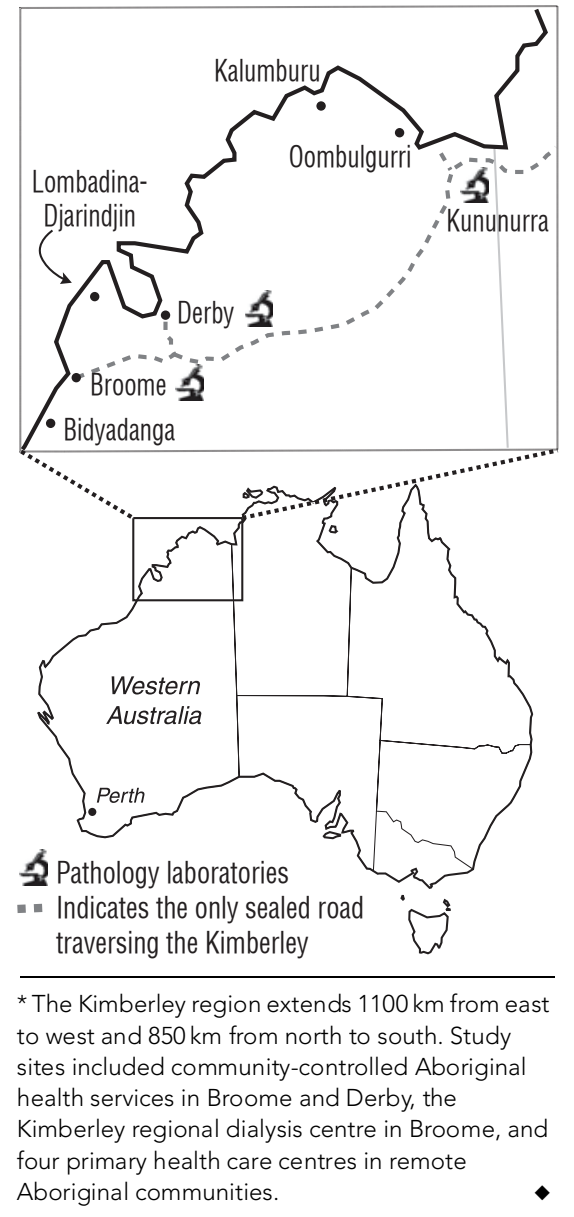

for the specimen to reach the laboratory varied from $30 \mathrm{~min}$ to 7 days.

\section{Ethical approval}

Ethical approval was obtained from The University of Western Australia Human Research Ethics Committee and the Western Australian Aboriginal Health Information and Ethics Committee. All study participants were provided with verbal and written information regarding the study, and informed written consent was obtained. If necessary, Aboriginal health workers provided cultural and language translations.

\section{Statistical analysis}

Data were analysed using SPSS, version 14.0 (SPSS Inc, Chicago, Ill, USA) and Stata, version 7.0 (StataCorp, College Station, Tex, USA) and are presented as mean and 95\% confidence intervals (CIs) unless otherwise stated. Concordance between POC and laboratory blood glucose values was assessed

\section{Comparison of subjects included and excluded from the analysis}

\begin{tabular}{lcc} 
& Included $(n=164)$ & Excluded $(n=36)$ \\
\hline Median age (range) in years & $44(16-65)$ & $47(14-86)$ \\
Women (\%) & $99(60 \%)$ & $27(75 \%)$ \\
Aboriginal and/or Torres Strait Islander (\%) & $150(91 \%)$ & $31(86 \%)$ \\
Pre-existing diagnosis of diabetes (\%) & $68(41 \%)$ & $23(64 \%)$ \\
Fasting at time of glucose measurement (\%) & $36(22 \%)$ & $7(19 \%)$ \\
Accu-Chek glucometer used (\%) & $35(21 \%)$ & $18(50 \%)$
\end{tabular}

\section{Concordance analysis of point-of-care (POC) and laboratory glucose results}
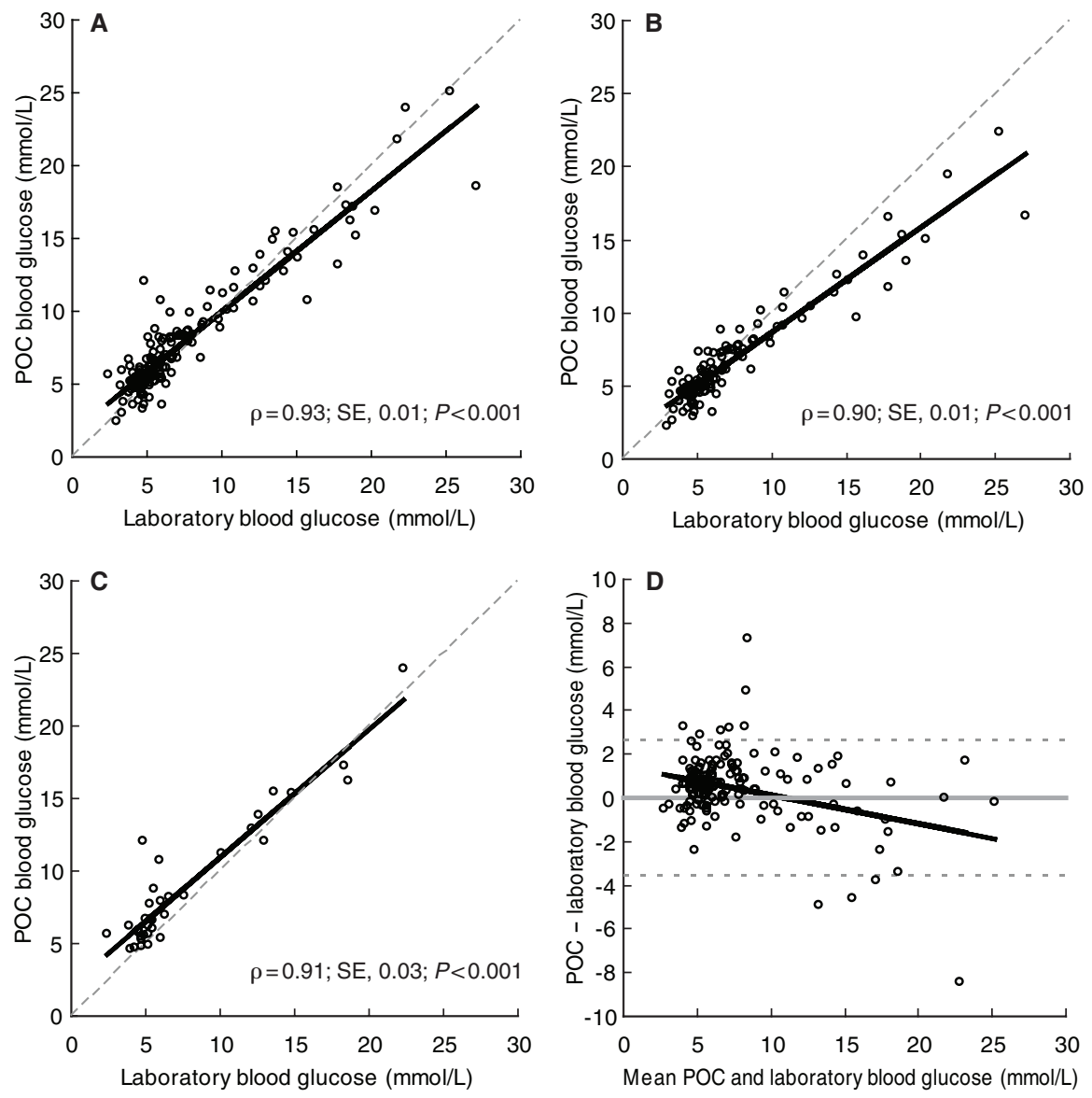

A: All unadjusted readings (plasma and whole blood levels) $(n=164) ; B$ : Capillary plasma MediSense readings converted to whole-blood levels $(n=129) ; C$ : Unadjusted Accu-Chek readings (whole blood levels) $(n=35)$. Dashed line in $A, B$ and $C=$ line of perfect concordance. $D$ : Difference plot (Bland-Altman analysis ${ }^{13}$ ) for all POC versus laboratory blood glucose measurements $(n=164)$. Dashed line in $D=95 \%$ limits of agreement.

by the technique of Lin, ${ }^{12}$ which measures the agreement between two methods. Mean difference (bias) and limits of agreement were determined using the techniques of Bland and Altman. ${ }^{13}$ POC capillary equivalence values for excluding and diagnosing diabetes were determined from receiver operating characteristics (ROC) curves, using laboratory values of $<5.5$ and $\geqslant 11.1 \mathrm{mmol} / \mathrm{L}$, respectively. Differences in proportions were determined using the twotailed Fisher's exact test. Multivariate linear regression analysis was used to determine whether patient or processing factors affected the relationship between POC and laboratory glucose levels. 


\section{DIABETES}

\section{RESULTS}

Two hundred participants were enrolled, and 164 included in the analysis. The reasons for exclusion were missing or inadequate tests in 29 participants, and more than 30 minutes elapsing between POC measurement and venipuncture in seven participants. Descriptive data on participants are listed in Box 2. Almost half of the excluded subjects (44\%) were assessed at one clinic, which happened to use an Accu-Chek glucometer, reducing the number of results for that glucometer.

Unadjusted POC blood glucose measurements and laboratory-measured venous blood glucose levels showed a high degree of concordance (Box 3A). The concordance was only marginally altered by stratifying the analysis by different glucometers and back-converting the MediSense POC results from plasma to whole blood (Box $3 \mathrm{~B}$ and Box 3C). The limits of agreement between $P O C$ and laboratory values are presented in Box 3D. Fasting POC blood specimens tended to have greater concordance with laboratory values $(\rho=0.96$; SE, 0.01 ; $P<0.001)$ compared with non-fasting specimens $(\rho=0.92$; SE, $0.01 ; P<0.001)$.

In a multivariate analysis, the MediSense glucometer and fasting at the time of blood glucose measurement were associated with less difference between the POC and laboratory glucose levels at clinically important values (0-12 mmol/L) and greater differences at higher values. These associations did not alter the explained variance of the model $\left(r^{2}=\right.$ 0.89 ), suggesting that this statistical relationship was of no clinical significance. The relationship between POC and laboratory glucose levels was not independently influenced by the time between POC glucose measurement and venipuncture, a pre-existing diagnosis of diabetes, or the time delay in collecting and processing the laboratory specimen.

The most clinically appropriate POC threshold value for excluding (venous blood glucose level, $<5.5 \mathrm{mmol} / \mathrm{L}$ ) or diagnosing diabetes (venous blood glucose level, $\geqslant 11.1 \mathrm{mmol} / \mathrm{L}$ ) was determined using ROC curves (Box 4). A POC reading of $<5.5 \mathrm{mmol} / \mathrm{L}$ had a sensitivity of $53.3 \%$, a specificity of $94.4 \%$, and a positive-predictive value of $88.9 \%$ for a venous value of $<5.5 \mathrm{mmol} / \mathrm{L}$. Sensitivity was increased to $61.9 \%$ (Fisher's exact test, $P=0.44$ ) in those subjects who had fasted before blood glucose measurement. The choice of POC glucometer had a significant influence on sensitivity (Accu-Chek, 29.4\%; MediSense, 60.3\%; P<0.05). However, none of these factors greatly influenced specificity. $\mathrm{POC}$ readings $\geqslant 12.2 \mathrm{mmol} / \mathrm{L}$ had
4 Receiver operating characteristics (ROC) curves for excluding and diagnosing diabetes
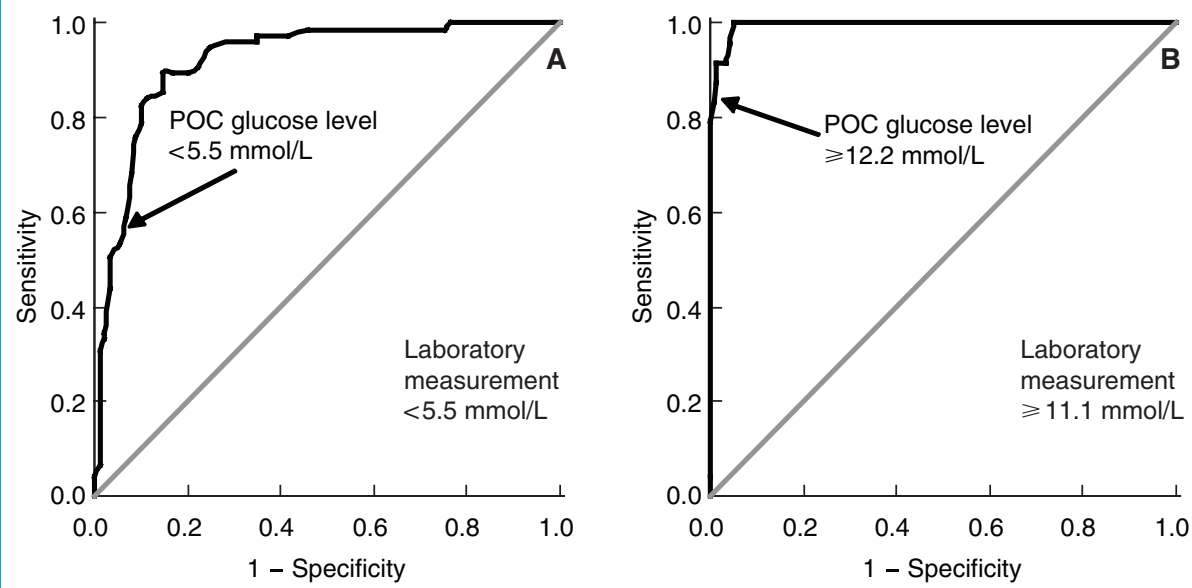

A: $R O C$ curves for excluding diabetes (laboratory-measured glucose level, $<5.5 \mathrm{mmol} / \mathrm{L}$ ). $B$ : $R O C$ curves for diagnosing diabetes (laboratory-measured glucose level, $\geqslant 11.1 \mathrm{mmol} / \mathrm{L}$ ).

5 Recommendations for the use of point-of-care glucose measurement in screening for and diagnosing diabetes in adults in the Kimberley

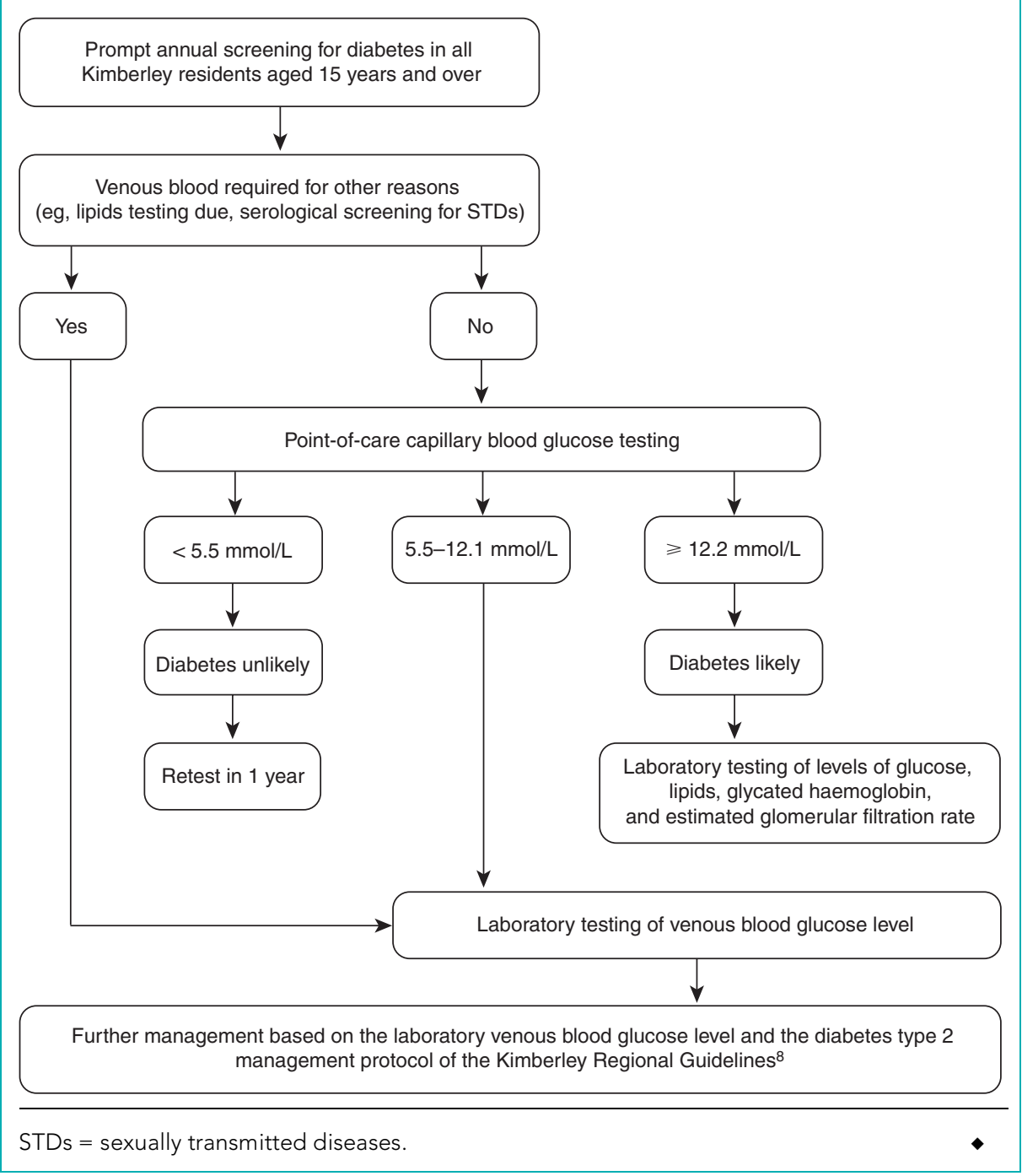


a sensitivity of $83.3 \%$, a specificity of $99.3 \%$, and a positive-predictive value of $95.2 \%$ for a venous value of $\geqslant 11.1 \mathrm{mmol} / \mathrm{L}$. In this setting, sensitivity was non-significantly reduced in fasted subjects $(75.0 \% ; P=0.54)$ and when using the MediSense glucometer (81.3\%; $P=$ 1.0) compared with not fasted (85.0\%) and using the Accu-Chek glucometer (87.5\%). Again, specificity was not greatly influenced by these factors.

\section{DISCUSSION}

Our study confirmed that obtaining venous glucose results in a timely manner in remote areas is very difficult. Some samples took 7 days to reach the laboratory, and on many occasions an appropriate venous sample did not arrive at all, leading to further delays and/ or missed diagnoses. Thus, for logistical reasons, relying solely on venous laboratory results remains a significant problem in the Kimberley and other remote areas.

POC values were generally marginally higher than venous samples at the lower end of the scale, but lower than venous results at the upper end of the scale (Box 3D). Nevertheless, this difference did not alter the diagnostic utility of POC capillary blood glucose measurement in this setting, as its high specificity showed. We also found that fasting POC blood glucose results are more sensitive than non-fasting results in excluding diabetes, and less sensitive in diagnosing it. However, it was reassuring that, regardless of whether the test was performed fasting or non-fasting, and irrespective of the glucometer used, specificity at both ends of the diagnostic range remained high.

In clinical practice, being able to either diagnose or exclude diabetes is far more important than concordance between POC and venous plasma levels across the range of glucose levels. The cut-off values for POC glucose levels and their diagnostic utility, however, require validation. ${ }^{10}$ While the guidelines of the Central Australian Rural Practitioners Association (CARPA) use cutoffs of 5.0 and $12.0 \mathrm{mmol} / \mathrm{L},{ }^{14}$ these are not evidence-based. Our results concurred with the World Health Organization recommendations of random POC plasma glucose levels $\geqslant 12.2 \mathrm{mmol} / \mathrm{L}$ for diagnosing diabetes and $<5.5 \mathrm{mmol} / \mathrm{L}$ for excluding diabetes. ${ }^{15}$ Assuming that the study population was typical of Kimberley residents undergoing screening for diabetes, using a POC glucose cut-off of $5.5 \mathrm{mmol} / \mathrm{L}$ to exclude diabetes will reduce the need for venipuncture by $24 \%$.
The national Australian guidelines for diabetes management recommend against using POC glucometers on the basis that they are not sufficiently accurate. ${ }^{6,7}$ Our study shows that, in real-world remote settings, POC measurement of blood glucose, with appropriate use of laboratory glucose measurement, is sufficiently accurate to be an important component of diabetes screening. In remote areas of Australia, POC glucose measurement can expedite the diagnosis of diabetes in high-risk, remote-dwelling populations and provide opportunities for immediate education and initiation of treatment.

Recommendations for the use of POC glucose measurement in the Kimberley developed from our study are outlined in Box 5.

\section{ACKNOWLEDGEMENTS}

Thanks to the staff and patients of Broome Regional Aboriginal Medical Service, Derby Aboriginal Health Service, Kimberley Satellite Dialysis Centre, and the Bidyadanga, Kalumburu, Lombadina-Djarindjin and Oombulgurri health care clinics for their participation in this study. Thanks also to Mena Lewis and Monica Frain for their assistance with data collection.

\section{COMPETING INTERESTS}

None identified.

\section{AUTHOR DETAILS}

Julia V Marley, PgDipSc, PgDipPolSt, PhD, Research Fellow ${ }^{1,4}$

Stephanie Davis, MB BS, Resident Medical Officer $^{2}$

Kerryn Coleman, MBBS, MPH, Resident Medical Officer $^{2}$

Bradleigh D Hayhow, BA(Hons), BMBS, Resident Medical Officer ${ }^{2}$

Greg Brennan, BNurs, Clinical Services Manager ${ }^{3}$ Jacki K Mein, MAE, FAChSHM, FAFPHM, Public Health Physician ${ }^{2}$

Carmel Nelson, MPH\&TM, FACRRM, FRACGP, Medical Director ${ }^{4}$

David Atkinson, MB BS, MPH, Medical

Coordinator, Medical Educator ${ }^{1,4}$

Graeme P Maguire, FRACP, MPH\&TM, PhD,

Physician ${ }^{5,6}$

1 The Rural Clinical School of Western Australia, The University of Western Australia, Broome, WA.

2 Western Australian Country Health Service Kimberley, Broome, WA.

3 Broome Regional Aboriginal Medical Service, Broome, WA.

4 Kimberley Aboriginal Medical Services Council, Broome, WA.

5 Department of Medicine, Cairns Hospital,

Cairns, QLD.

6 Infectious Diseases Division, Menzies School of Health Research and Charles Darwin University, Darwin, NT.

Correspondence: Julia.Marley@uwa.edu.au

\section{REFERENCES}

1 Daniel M, Rowley KG, McDermott R, et al. Diabetes incidence in an Australian Aboriginal population. An 8-year follow-up study. Diabetes Care 1999; 22: 1993-1998.

2 Martin DD, Shephard MD, Freeman H, et al. Pointof-care testing of $\mathrm{HbA}_{1 \mathrm{c}}$ and blood glucose in a remote Aboriginal Australian community. Med $J$ Aust 2005; 182: 524-527.

3 Dunstan DW, Zimmet PZ, Welborn TA, et al. The rising prevalence of diabetes and impaired glucose tolerance: the Australian Diabetes, Obesity and Lifestyle Study. Diabetes Care 2002; 25: 829-834.

4 The Diabetes Control and Complications Trial Research Group. The effect of intensive treatment of diabetes on the development and progression of long-term complications in insulin-dependent diabetes mellitus. N Engl J Med 1993; 329: 977-986.

5 UK Prospective Diabetes Study (UKPDS) Group. Intensive blood-glucose control with sulphonylureas or insulin compared with conventional treatment and risk of complications in patients with type 2 diabetes (UKPDS 33). Lancet 1998; 352: 837-853.

6 The Royal Australian College of General Practitioners and Diabetes Australia. Diabetes management in general practice. 12th ed. Melbourne: RACGP and Diabetes Australia, 2006/7. http:// www.racgp.org.au/guidelines/diabetes (accessed Apr 2007).

7 The Australian Centre for Diabetes Strategies. National evidence based guidelines for the management of type 2 diabetes mellitus. Sydney: Prince of Wales Hospital and National Health and Medical Research Council of Australia, 2001. http:// www.diabetesaustralia.com.au/_lib/doc_pdf/ NEBG/CD/Part3-CaseDetection-311201.pdf (accessed Dec 2006).

8 Kimberley Aboriginal Medical Services Council (KAMSC) and WA Country Health Service (WACHS) - Kimberley. Chronic disease protocols. Diabetes type II. http://www.kamsc.org.au/cd_protocols.html (accessed Apr 2007)

9 Cohen M, Boyle E, Delaney C, et al. A comparison of blood glucose meters in Australia. Diabetes Res Clin Pract 2006; 71: 113-118.

10 Colagiuri S, Sandbaek A, Carstensen B, et al. Comparability of venous and capillary glucose measurements in blood. Diabet Med 2003; 20: 953-956.

11 Kruijshoop M, Feskens EJ, Blaak EE, et al. Validation of capillary glucose measurements to detect glucose intolerance or type 2 diabetes mellitus in the general population. Clin Chim Acta 2004; 341: 33-40.

12 Lin LI. A concordance correlation coefficient to evaluate reproducibility. Biometrics 1989; 45: 255268.

13 Bland JM, Altman DG. Statistical methods for assessing agreement between two methods of clinical measurement. Lancet 1986; 1: 307-310.

14 Ewald D, editor. CARPA standard treatment manual. A clinical manual for primary health care practitioners in remote and rural communities in Central and Northern Australia. 4th ed. Alice Springs: Central Australian Rural Practitioners Association, 2003.

15 World Health Organization. Definition, diagnosis and classification of diabetes mellitus and its complications. Report of a WHO Consultation. Part 1. Diagnosis and classification of diabetes mellitus. Geneva: WHO, 1999. Report No. WHO/NCD/ NCS/99.2. http://whqlibdoc.who.int/hq/1999/ WHO_NCD_NCS_99.2.pdf (accessed Jan 2007). 